\title{
On discriminating temporal relations: Is it relational?
}

\author{
LEON R. DREYFUS \\ Vassar College, Poughkeepsie, New York \\ J. GREGOR FETTERMAN \\ Indiana University-Purdue University, Indianapolis, Indiana \\ D. ALAN STUBBS \\ University of Maine, Orono, Maine \\ and \\ SUSAN MONTELLO \\ Vassar College, Poughkeepsie, New York
}

\begin{abstract}
Pigeons were presented on each trial with a pair of keylight stimuli that varied in duration. One of two subsequent choices was reinforced, depending on which of the two stimuli was longer. For some pairs, the duration of one stimulus was predictive of relative duration, but for other pairs, absolute duration was unpredictive. Choice responses depended on relative differences between the stimuli, but were also controlled to some degree by absolute duration of the second member of the pair. Individual differences in control by absolute and relative duration were evident. Those pigeons whose behavior was most influenced by absolute duration showed poorer transfer to a different set of duration pairs.
\end{abstract}

A resurgence of interest in comparative cognition (e.g., Roitblat, 1987; Roitblat, Bever, \& Terrace, 1984) has been accompanied by the development of an array of procedures for studying the abilities of nonhuman animals. New procedures have been developed (e.g., Olton \& Samuelson, 1976) and standard procedures have been adapted (e.g., Wright, Santiago, Sands, Kendrick, \& Cook, 1985) for use with nonhumans. Over the last several years, we have undertaken a series of investigations of the performance of pigeons on a temporal pair-comparison procedure (Dreyfus, Fetterman, Smith, \& Stubbs, 1988; Fetterman \& Dreyfus, 1987; Fetterman, Dreyfus, \& Stubbs, 1989). The method of pair comparison (Stevens, 1951) involves the presentation of pairs of stimuli with the observer reporting differences with respect to some dimension between the stimuli of the pair. In our adaptation of this procedure, pigeons are required to respond on the basis of the relative duration of successively presented visual stimuli. Several basic findings have emerged from this research program. First, responding depends on the

This research was supported in part by NSF REU 8901020 to Vassar College. J. Gregor Fetterman was supported during preparation of this paper by NSF Grant BNS-9021562. Portions of these data were presented at the meeting of the Eastern Psychological Association, Boston, 1991, and at the Dalhousie Conference on Cognitive Aspects of Stimulus Control, Halifax, 1989. Correspondence should be addressed to $L$. $R$. Dreyfus, Department of Psychology, Vassar College, Poughkeepsie, NY 12601; e-mail: dreyfus@vaxsar.vassar.edu. relative, not the absolute, difference between durations (Fetterman \& Dreyfus, 1986, 1987). This finding is consistent with results using other timing procedures (e.g., Stubbs, 1968) and with Weber's law. Second, a bias to report one duration of the pair as being longer than the other changes systematically with the absolute value of the sum of the two durations (Fetterman \& Dreyfus, 1987). Third, pigeons are capable of discriminating temporal relations over a wide range of durations and duration ratios but with a decrease in accuracy as duration range increases (Dreyfus et al., 1988). Fourth, pigeons are equally accurate on the task whether it involves a discrimination of one duration as longer or shorter than the other or it involves a discrimination of whether a duration ratio (e.g., second/first duration) is greater or less than some criterion ratio, for example, 4:1 (Fetterman et al., 1989).

The potential value of these studies stems from two important considerations. First, the pair-comparison method differs in a fundamental way from most other methods used to study time perception in nonhumans (see reviews by Richelle \& Lejeune, 1980; Stubbs, 1979). For most of these procedures, the consequences of responding are arranged with respect to some fixed temporal criterion (e.g., the time of food on a fixed-interval schedule, the cutoff duration on a psychophysical task). By contrast, with the method of pair comparison, choice responses are reinforced on the basis of the relation between successively presented durations, each of which may change 
from trial to trial. The second consideration is that this task, in addition to its usefulness in addressing questions about time perception, lends itself to addressing questions about relational learning and memory in nonhumans. The present investigation focuses on the relational aspects of performance on this task. Although the study of relational learning by nonhumans has a long history (e.g., Lawrence \& DeRivera, 1954), recent research in this area has been stimulated by a general interest in the cognitive processes of nonhumans and a particular interest in their conceptual abilities (e.g., Premack, 1983).

Nominally, the duration-comparison task requires responding on the basis of stimulus relations, but it became evident in our earliest studies that many choices could be based on the absolute duration of one of the stimuli of a pair rather than on the relation between durations of the pair. A correlation between absolute and relative duration allowed subjects to base choices on a single duration and still perform at a level considerably greater than chance. This correlation is shown in Figure 1 for several different procedures for determining durations and pairs. The scatterplots depict the relation between one duration of the pair and the ratio of the two durations. Since the correct choice response and reinforcement depend on which duration of the pair is longer, a vertical dotted line has been placed at the point at which the two durations are equal. For problems to the left of this line, the first duration is longer than the second, and for problems to the right, the second duration is longer than the first. Different numbers of points in each panel reflect the different numbers of unique problems under each method for determining durations.

The left panel of Figure 1 shows the relation between absolute and relative duration for our earliest experiments with the duration-comparison procedure (Fetterman \& Dreyfus, 1986). Under this procedure, 16 different pairs resulted from pairwise combination of four different durations. In training, the equal pairs were not employed, leaving only 12 unique pairs. For these 12 pairs, it is evident that the longest second duration was always longer than its pair member whereas the shortest second duration was always shorter than its pair member. Therefore, on the basis of absolute duration alone, it would be possible to respond correctly on all trials involving these durations. The intermediate durations were not perfectly correlated with duration ratio, but nevertheless, on two thirds of the trials the second longest duration was longer than its pair member and the third longest duration was shorter than its pair member. This relation between second duration and duration ratio is reflected in the upward slope of points; essentially, as the second duration gets longer, it is more likely to be longer than the first. The first duration is also correlated with duration ratio but in the opposite direction. Because of the correlation between absolute and relative duration, an absolute response strategy (i.e., responding on the basis of whether one of the durations of the pair was shorter or longer than a criterion) could produce an accuracy level as high as $83 \%$ on the training problems shown in the left panel of Figure 1. Analyses of performance on this task, including that following the introduction of novel probe pairs or an interstimulus interval, indicated that there was indeed substantial control by absolute duration (Fetterman \& Dreyfus, 1986).

In a subsequent experiment, the procedure for determining durations and duration pairs was changed in order to reduce the confounding of absolute and relative duration (Fetterman \& Dreyfus, 1987). Under the modified procedure, first and second durations were chosen independently and probabilistically from a set of 32 different durations. The middle panel of Figure 1 shows the relation between absolute and relative duration that resulted. Several differences from the left panel are evident. Under the modified procedure, there were many more unique problems and the ranges of durations and duration ratios were larger. The correlation between absolute and relative duration is, however, still evident. The top row and bottom row of points show that the longest durations were always longer than, and the shortest durations were always shorter than, their pair members. The distribution of points is still characterized by an overall positive slope. Because of the larger number of intermediate durations, however, the maximum level of accuracy that could be achieved by responding solely on the basis of absolute duration was reduced to $76 \%$. In addition, it should
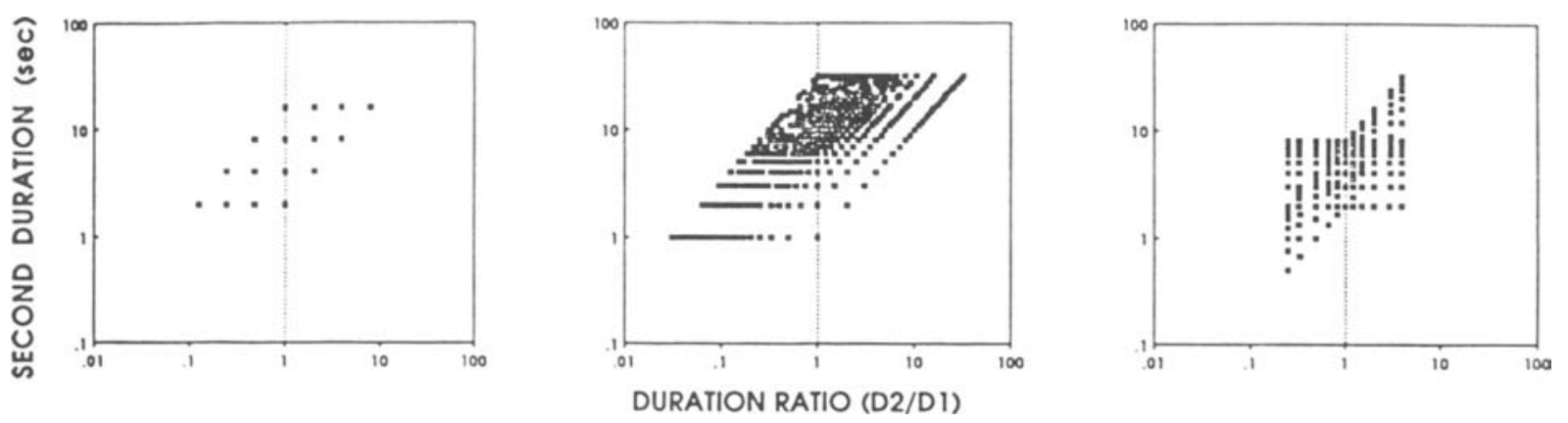

Figure 1. Scatterplots of the relation between the second duration of a pair and the ratio of the durations for three different procedures for determining durations and pairs. 
be noted that discriminating absolute durations would be more difficult under the modified procedure since durations were relatively closer together. Analyses of performance under this method of determining durations showed that behavior was controlled to some extent by both absolute and relative duration (Dreyfus et al., 1988).

The present experiment was conducted to evaluate further the role of absolute and relative duration on temporal pair-comparison performance. First, the procedure was further modified to reduce the confounding between $a b$ solute and relative duration, and second, a transfer test was employed in which absolute response strategies could be identified. The right panel of Figure 1 shows the distribution of problems used in the current experiment (details of the procedure for determining durations will be presented later). The distribution of problems represented by the scatterplot is similar to those shown in the prior two panels in that there is an overall positive slope. There is an important difference, however. In the current set of problems, there is a subset of durations, the rectangle bounded by $2 \mathrm{sec}$ and $8 \mathrm{sec}$, for which absolute duration is unpredictive of duration ratio, that is, it is equally likely that a duration of this set is longer or shorter than its pair member. If accurate performance on the task requires that the correlation between absolute and relative duration extend throughout the problem set, then discrimination performance might be poorer on those problems for which absolute duration is not predictive of relative duration. As a result of the change in the method of determining durations, the maximum accuracy that could be achieved by nonrelational means was reduced further to $68 \%$.

The presence of responding on the basis of absolute and relative duration cues and the consequences of such response patterns were evaluated further by transferring the pigeons to a new distribution of problems. Under the initial distribution of problems used in this experiment (right panel of Figure 1), durations in the 2-8-sec range were unpredictive of relative duration whereas those less than $2 \mathrm{sec}$ or greater than $8 \mathrm{sec}$ were highly correlated with relative duration. Some of the durations that had been informative under the initial distribution became uninformative under the new distribution of problems. Reliance on absolute cues should produce lower accuracy in performance on the new distribution and, in particular, should produce systematic errors on those problems for which absolute duration is no longer informative.

\section{Method}

\section{Subjects}

Four experimentally naive White Carneau pigeons served as subjects. These pigeons were maintained at approximately $85 \%$ of their free-feeding weights and were given unlimited access to water and grit in their home cages.

\section{Apparatus}

The experiment was conducted in two sound-insulated, ventilated pigeon chambers with work spaces measuring $32 \mathrm{~cm}$ high $\times 34 \mathrm{~cm}$ wide $\times 33.5 \mathrm{~cm}$ deep. Three keys were accessible through $2-\mathrm{cm}$ circular openings in the work panel, with the center of the open- ings spaced $6.3 \mathrm{~cm}$ apart and $23 \mathrm{~cm}$ above a wire-mesh floor. A force of approximately $0.15 \mathrm{~N}$ was required to operate the keys. A solenoid-operated grain feeder could be accessed through a $5.5-\mathrm{cm}^{2}$ opening centrally located below the keys; the bottom of this opening was $9 \mathrm{~cm}$ above the floor. Masking noise was provided through a speaker in the chamber. Experimental events were scheduled and recorded by laboratory computers and interfaces in an adjacent room.

\section{Procedure}

Pretraining for the pigeons began with magazine training followed by an autoshaping procedure (Brown \& Jenkins, 1968) in which one of the side keys was lit by red or green light on each trial. The position and color of the lit key changed irregularly over trials. Following $6 \mathrm{sec}$ of keylight illumination, the feeder was raised for $3 \mathrm{sec}$. A peck to the lit side key turned it off and produced food immediately. During food presentation, all lights in the chamber were off except for the light in the food hopper. Food presentation was followed by an intertrial interval (ITI) that averaged $30 \mathrm{sec}$ and during which the chamber was completely dark. The next trial began with illumination of the keylight and houselight. Each pigeon received 60 trials per day on this procedure until a peck was recorded to the lit key on at least $75 \%$ of the trials on 2 successive days.

After the first criterion was met, the autoshaping procedure was modified so that only the center key was illuminated by white light on each trial. The pigeons were continued on this procedure until the same criterion was met. Finally, these two autoshaping procedures were combined so that each trial began with illumination of the center key by white light for a maximum of $6 \mathrm{sec}$. The offset of the center-key stimulus was followed by illumination of one of the side keys by red or green light. A peck to the lit center key turned off the white light and illuminated the side key. If there were no side-key responses, the side key remained lit for $6 \mathrm{sec}$ and was followed immediately by $3 \mathrm{sec}$ of food. A peck to the lit side key turned off the light and produced food immediately. The pigeons remained on this procedure until the same criterion was met.

Following pretraining, the pigeons were placed directly on the duration-comparison procedure. Each trial began with the illumination of the center key by white light. A single peck to the key changed the illumination from white to red and began the first duration. The center key remained red for a period of time that varied from trial to trial, and then the center key illumination changed from red to green, beginning the second duration. Pecks to the center key during the first or second duration were recorded but had no scheduled consequences. At the end of the second duration, the center key was darkened and the side keys were illuminated, one by red and one by green light. The location of red and green changed irregularly over trials so that each appeared about equally often on the right and left. On a trial on which the first (red) duration was longer than the second (green), a peck to the red side key was reinforced. If the second duration was longer than the first, a peck to the green side key was reinforced. Pecks to the incorrect side key produced a 15-sec blackout during which all lights in the chamber were off. Reinforcement consisted of 3-sec access to mixed grain and was followed by a $12-\mathrm{sec}$ blackout. A noncorrection procedure was used, and the next trial began with illumination of the center key.

Duration pairs were constructed by first designating the seven integer values from 2 through $8 \mathrm{sec}$ as "base" durations. One of these base durations served as one member of the pair on each trial. The other member of the pair was determined by multiplying or dividing the base duration by factors of 2,3 , and 4 . This method produced six pair members for each of the seven base durations (e.g., a base duration of $6 \mathrm{sec}$ was paired with durations of 1.5, $2,3,12,18$, and $24 \mathrm{sec}$ ). Pairing each of the seven base durations with each of its possible pair members produced 42 problems. Allowing each base duration to appear as first or second duration in a pair increased this number to 84 , and removing redundant pairs 
reduced the number to 74 unique pairs. During training, sessions consisted of 148 trials, two repetitions of each of the 74 unique pairs. In this and all other phases of the experiment, the order of the problems was changed daily.

After all of the pigeons had reached a criterion of $85 \%$ correct on five consecutive days, the number of trials per session was increased to 222, 3 repetitions of each unique pair, and reinforcement was made available on a random one half of the trials. On correct but nonreinforced trials, pigeons' choices produced a $15-\mathrm{sec}$ blackout. After five sessions on this procedure, reinforcement availability was further reduced to a random one third of trials. When performance stabilized on this schedule, novel-duration probe pairs were introduced.

Probe pairs were arranged by multiplying and dividing each base duration by factors of $1.5,1.2$, and 1.0, the last of these multipliers creating pairs with equal first and second durations. Removing redundant pairs and allowing each problem with equal first and second durations to appear twice in the set resulted in 64 novel probe pairs. During probe testing, there were 212 trials per day; 148 of those consisted of training pairs (two repetitions of the training set) with reinforcement available on a random one half of these trials. The remaining 64 trials consisted of the probe pairs on which no reinforcement was available.

Following 12 sessions of exposure to novel probe pairs, the pigeons were given a 2-month layoff, after which they were returned for 13 sessions to conditions identical to those during the introduction of novel probes. Conditions were then changed so that reinforcement was available on a random one half of all trials except those on which the two durations of the pair were equal. After 10 sessions, the distribution of problems was changed. For the new distribution, duration pairs were calculated in the same way as be fore but using seven different base durations, 6-12 sec rather than 2-8 sec. The composition of the problem set was comparable to that of the earlier range except that there were 228 daily trials rather than 212 because there were fewer redundant pairs in the 6-12-sec range. Reinforcement was potentially available on a random one half of all trials except those on which the two durations of the pair were equal. The pigeons were tested under this new range for 18 to 20 sessions. This new range set up an interesting transfer test insofar as under the 2-8-sec range, durations of 9-12 sec would always be longer than their pair members. Thus, under an absoluteduration strategy, these durations should always be reported as being longer than their pair members. Under the 6-12-sec base range, however, durations of 9-12 $\mathrm{sec}$ were equally likely to be longer or shorter than their pair members. Thus, following the change to the 6-12-sec range, an absolute strategy based upon durations of 9-12 sec would produce errors on $50 \%$ of these problems.

\section{RESULTS}

The current procedure for determining durations reduced the predictive relation between absolute and relative duration, but the right panel of Figure 1 makes it clear that some degree of predictability remained. The extent to which absolute duration was predictive of duration ratio is summarized in Figure 2. This figure shows the proportion of trials on which the second duration (D2) is longer than the first duration (D1) for three different categories of D1 and D2. Duration categories were arranged to include approximately equal numbers of problems in each category. Trials on which the durations were equal were not included in the analysis, so the proportion of trials on which D1 was longer than D2 is the complement of the proportions shown. This figure shows that when one of the durations was in the short category, it was likely

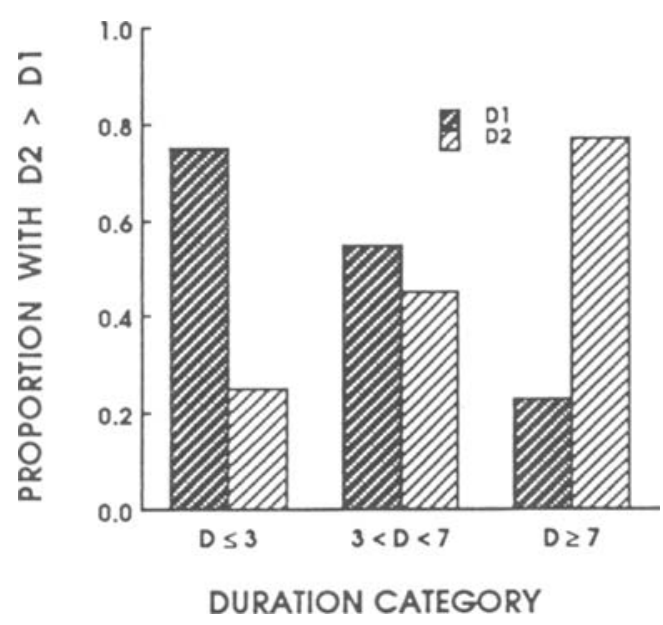

Figure 2. Proportion of trials on which the second duration (in seconds) of the pair is longer than the first duration for three absoluteduration categories.

to be shorter than its pair member and when it was in the long category, it was likely to be longer than its pair member. A duration in the middle category was about as likely to be longer or shorter than its pair member. The slight asymmetry in this category resulted from the manner in which redundant problems were removed from the set.

The extent to which discrimination varied as a function of absolute and relative duration can be assessed by comparing performance across the three categories shown in Figure 2. If there were effects of absolute duration, Figure 2 would lead us to expect that a duration in the short category would more likely be reported as shorter than its pair member, whereas a duration in the long category would more likely be reported as longer than its pair member.

Figure 3 shows how the predictive relations summarized in Figure 2 affected performance. In this figure, the probability of a green-key response, reporting D2 as longer than D1, is plotted as a function of duration ratio for each of the D1 and D2 categories shown in Figure 2. For data plotted in this format, accuracy is reflected in the slope of the curve, with a steeper the curve indicating more accurate performance, and bias is indicated by a separation of the curves. Performance on all problems is shown in both left and right panels, but the problems in the left panels are segregated according to D1 categories and those in the right panels according to D2 categories. One reason for displaying the data by both D1 and D2 categories was to determine if choices were influenced by the absolute value of D1, D2, or both. Such an effect would appear as a separation of the curves in each panel. If, for example, a pigeon was responding on the basis of the absolute value of $\mathrm{D} 1$ and in accord with the correlation shown in Figures 1 and 2, the filled circles in the left panels would be displaced above the symbols for the intermediate category and the unfilled circles would be displaced below the points for the intermediate category. Such 


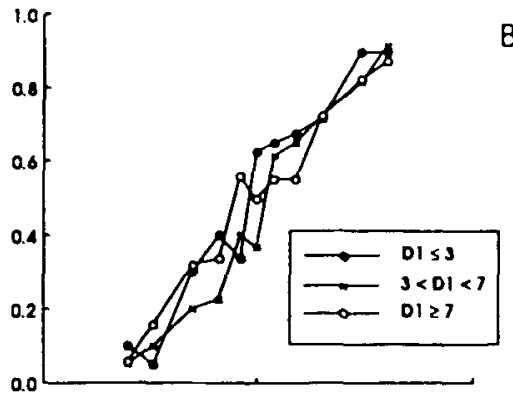

B50
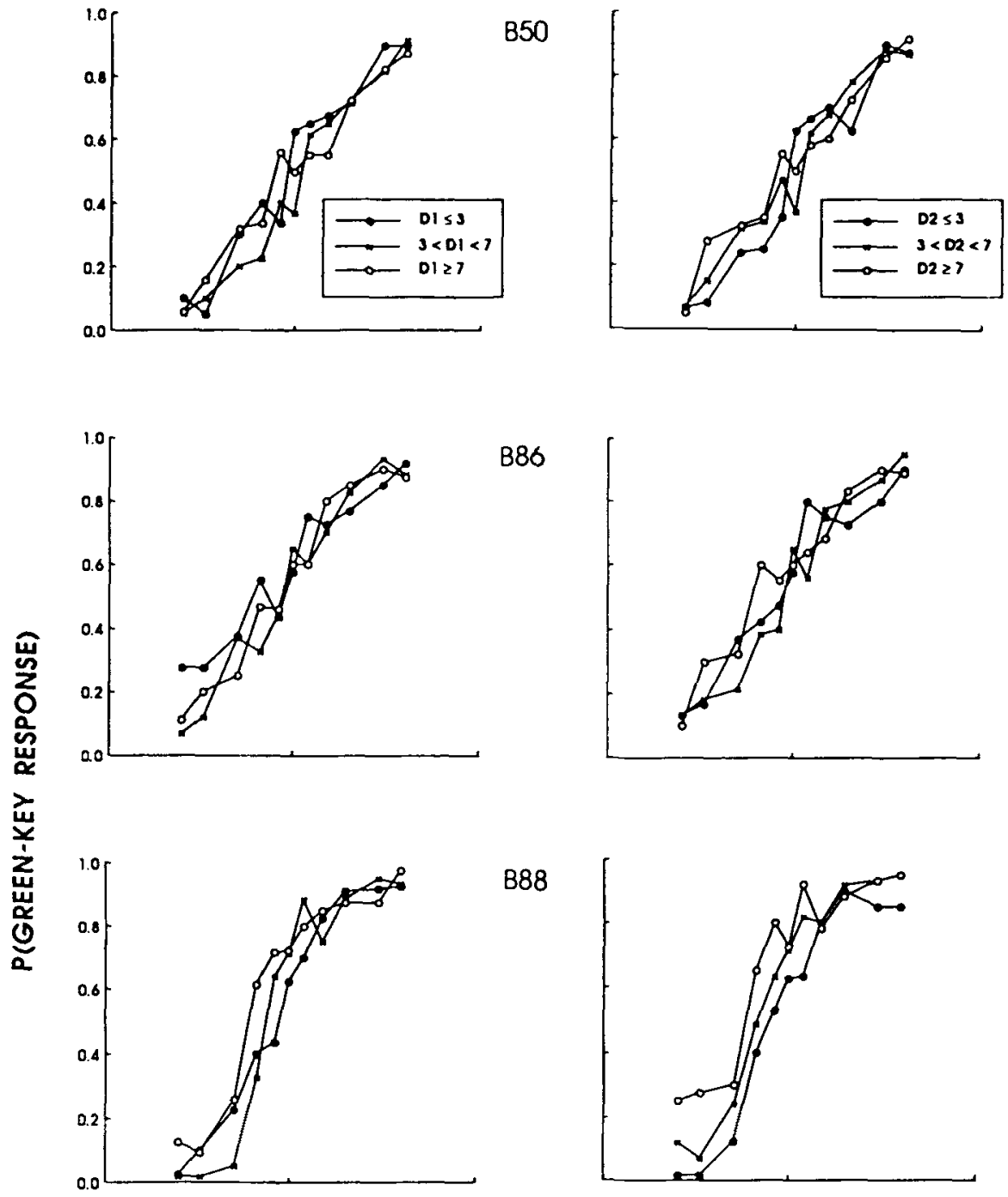

B86

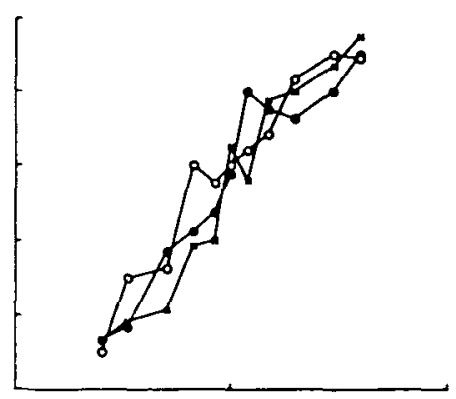

888
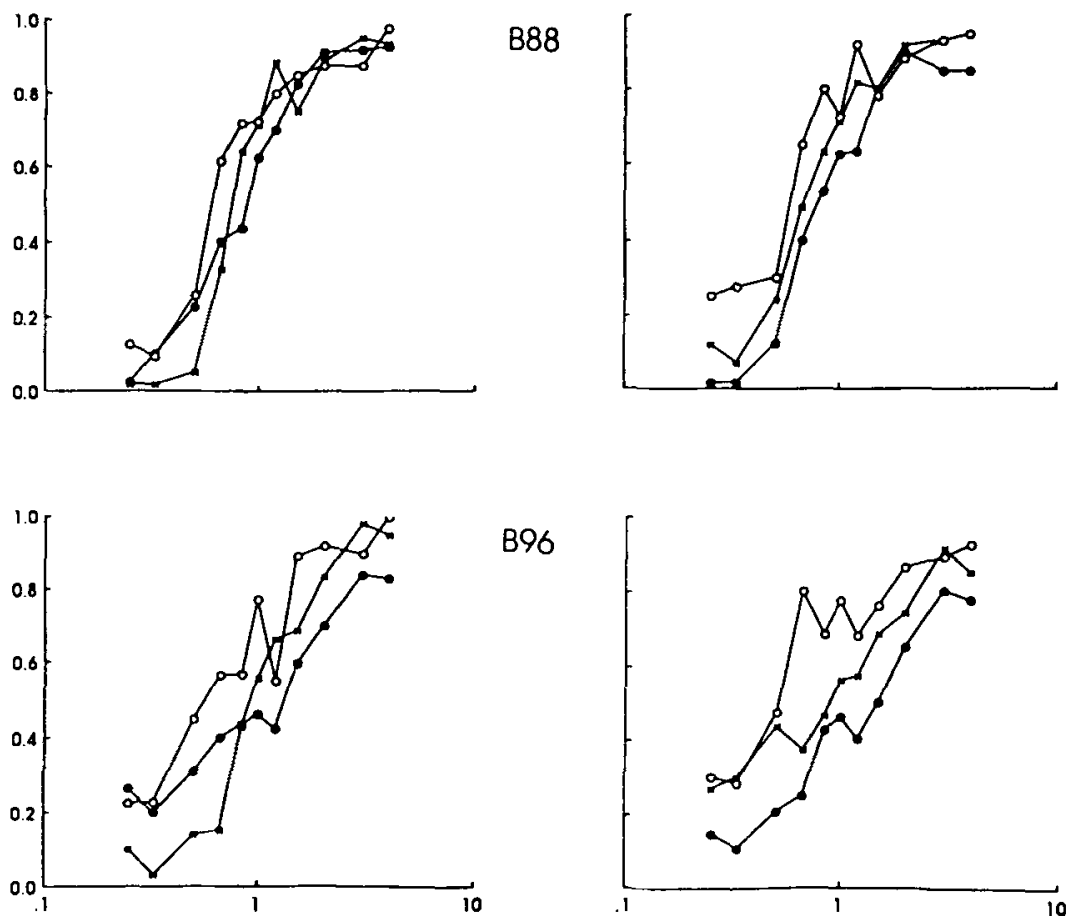

B96

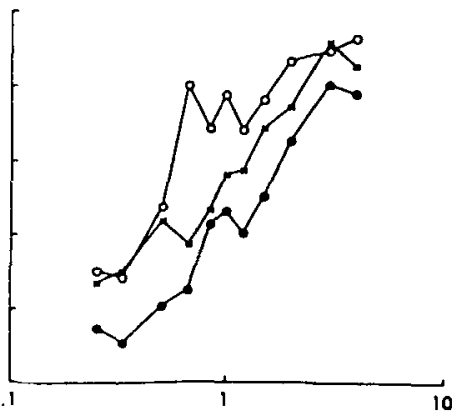

DURATION RATIO (G/R)

Figure 3. Probability of a green-key response as a function of duration ratio for each of 4 pigeons with separate functions plotted for each of three first-duration (in seconds) categories in the left panels and for each of three second-duration categories in the right panels. (These data were accumulated over the final 10 sessions of the condition.) 
a displacement of points would place the curve for the shortest D1s to the left of the curve for the intermediate durations and the curve for the longest D1s to the right. Responding on the basis of the absolute value of D2 would produce the opposite pattern of displacements in the right panels: the curve for the longest D2s (unfilled circles) would be displaced to the left and the curve for the shortest D2s (filled circles) would be displaced to the right.

The data in this figure indicate that performance was controlled by both absolute and relative duration; whereas control by absolute duration varied across subjects, control by duration ratio was present for all 4 subjects. For pigeons B50 and B86, the relatively small separation among the curves offers little evidence that responding differed over absolute-duration categories of D1 or D2. By contrast, B88 shows a slight absolute-duration effect and B96 a more substantial absolute-duration effect. In addition, the data in this figure show that when absolute duration affected performance, it was D2 that exerted control. For both B88 and B96, the absolute duration of the first stimulus had an effect opposite of that expected on the basis of the correlations shown in Figures 1 and 2 . Those figures suggested that short values of D1 should increase the probability of reporting green longer, but the left panels of this figure show that this was generally not the case. The absolute effect for D2, where it is evident, is consistent with the expectations based on Figures 1 and 2. At a given duration ratio, the highest probabilities of reporting green longer for both B88 and B96 occurred when green was in the long category and the lowest probabilities occurred when green was in the short category.

Figure 3 also demonstrates control by relative duration. A relational component of performance is suggested insofar as the probability of making a green-key response in the intermediate-duration categories changes as an orderly function of duration ratio. Since D2s in this category are base durations and not predictive of duration ratio, accurate discrimination in this category must either be based on $\mathrm{D} 1$ or on relative duration. The data do not support the first possibility. For at least 2 pigeons, B50 and B86, there was no appreciable control by absolute duration, and for the other 2 pigeons, control by absolute duration seemed to be limited primarily to D2. Thus, even for those pigeons who show an absolute-duration effect, when D2 is in the intermediate category it seems likely that choice responses are based on the temporal relation between durations. In addition, the overall level of accuracy provides further evidence that performance was based on relative duration. Average accuracy for the 4 pigeons was $80 \%$; the maximum achievable by nonrelational means, $68 \%$, is more than three standard deviations lower than the obtained average.

The data in Figure 3 also suggest that there were differences in accuracy over absolute-duration categories (e.g., for several pigeons, the curve for the intermediate Dl category appears steeper than the other curves). These differences in accuracy as well as differences in bias can be evaluated more explicitly by calculating summary measures of performance. Figure 4 shows $A^{\prime}$, a nonparamet-
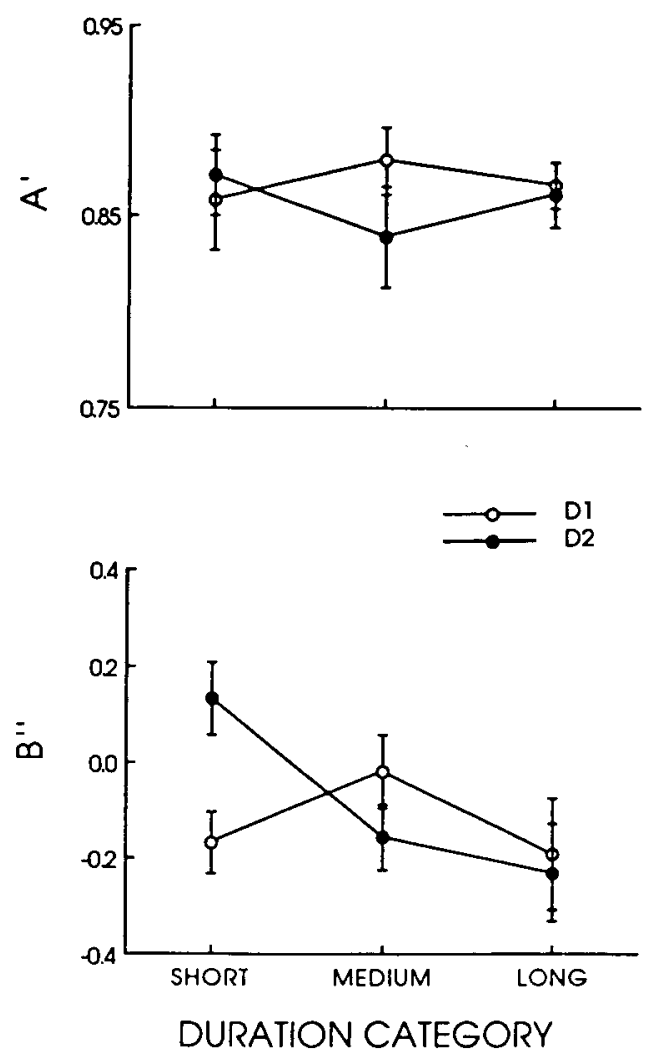

Figure 4. Average sensitivity, $A^{\prime}$, and bias, $B^{\prime \prime}$, for problems with first or second durations falling in one of three different absoluteduration categories. (These measures were computed from data accumulated over the final 10 sessions of the condition and were averaged over pigeons. Error bars represent standard errors of the means. Note the restricted range of $A^{\prime}$ values represented on the axis.)

ric index of sensitivity, and $\mathrm{B}^{\prime \prime}$, a nonparametric index of bias, for each category of D1 and D2 (see Grier, 1971, for a general treatment of these measures and Dreyfus et al., 1988, for a discussion of their use with similar data). $A^{\prime}$ is highly correlated with accuracy but is independent of changes in accuracy resulting from shifting biases. $B^{\prime \prime}$ reflects systematic tendencies to strike either choice key more frequently. Positive values of B" indicate a bias to peck the red key (reporting the first duration as longer), negative values indicate a bias to strike the green key (reporting the second duration as longer), and $\mathrm{a}^{\prime \prime}$ of zero indicates no bias. The upper panel of this figure shows that sensitivity for D1s and D2s was nearly identical for short and long absolute-duration categories. On the other hand, sensitivity was higher for problems with D1s in the intermediate category than for those with D2s in this category. This difference is consistent with the conclusion drawn from Figure 3 that choices on some problems were controlled by absolute values of the D2 of the pair. D1s in the intermediate category were paired most often with D2s in one of the extreme categories. If the pigeons were responding on the basis of D2 alone, one would expect higher accuracy in this category. For problems with D2 in the intermediate 
category, on the other hand, responding on the basis of absolute duration would produce lower accuracy since D2 was largely unpredictive of duration ratio.

The bottom panel shows that the way in which bias changed over absolute-duration categories differed depending on whether problems were segregated by D1 or D2. With problems categorized by D1, there was a bias to report D1 longer for problems with a short or long D1 and little bias for problems with a medium-length D1. With problems categorized by D2, bias changed systematically from a bias to report D1 longer for short D2s to a bias to report D2 longer for long D2s. These changes in B" over absolute-duration categories also show that when a choice was made on the basis of absolute value, it was typically based on D2. Changes in B" over D2 categories are completely consistent with the relation of D2 and duration ratio shown in Figures 1 and 2, whereas the changes in B" over D1 categories are not consistent with the relation of D1 and duration ratio.

The results of the transfer from the 2-8-sec range to the 6-12-sec range are informative about the extent to which performance was influenced by the absolute values of individual pair members. Figure 5 shows discrimination performance on the subset of problems that would be most affected by responding on the basis of absolute duration, D2s from 9 through $12 \mathrm{sec}$, compared with performance on all other problems. As before, slopes of the functions reflect accuracy and separation of the curves indicates bias. This figure reveals individual differences in the extent to which performance differed on the two subsets of problems. Performance on the two subsets is relatively similar for B50 and B86, although there is a difference for B86 at small ratios. The differences in performance on the problem subsets are more extensive and more systematic for B88 and B96. For the 9-12-sec D2s, both of these pigeons show a higher probability of reporting green longer when the ratio of the durations was less than 1.0 .

Summary measures of sensitivity, $\mathbf{A}^{\prime}$, and bias, $\mathbf{B}^{\prime \prime}$, were calculated for the two subsets of problems and compared with one-tailed $t$ tests. A ${ }^{\prime}$ was significantly lower under the 9-12-sec range than for all other problems [ $t(6)$ $=-1.98, p<.05]$. The difference in $\mathrm{B}^{\prime \prime}$ was not significant $[t(6)=-1.51, p=.09]$.

The extent to which performance differed under the two base duration ranges is summarized in Figure 6 . This figure shows average sensitivity, $A^{\prime}$, and bias, $B^{\prime \prime}$, for the 2-8-sec and 6-12-sec base ranges at each of three D2 categories. The pattern of changes in $\mathrm{A}^{\prime}$ over D2 categories is similar for both base ranges; accuracy is higher, however, for the 2-8-sec range. The lower panel shows that under the two base ranges there are similar changes in bias over D2 categories. This pattern of bias, a red-key bias for problems with short D2s and a green-key bias for problems with long D2s, is consistent with the relation between absolute duration and duration ratio presented in Figures 1 and 2.
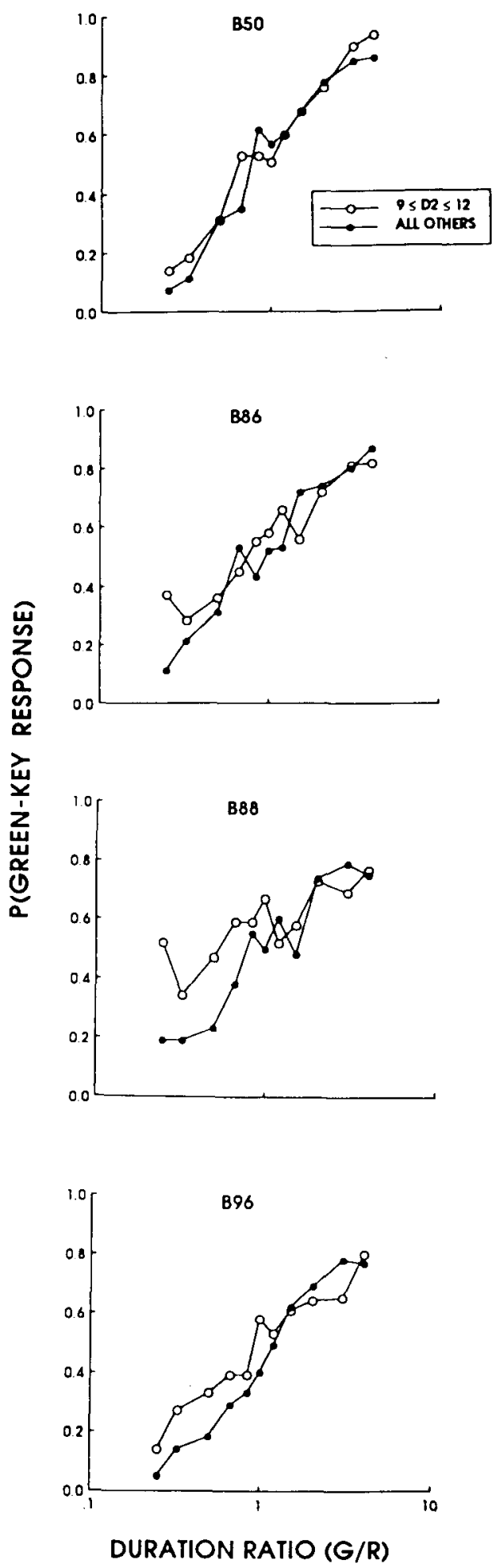

Figure 5. Probability of a green-key response as a function of duration ratio for each of four pigeons after being transferred to the 6-12-sec base range. (Separate functions are plotted for problems in a restricted second-duration category and for all other problems. These data were accumulated over the first 10 sessions of exposure to the 6-12-sec base range.) 

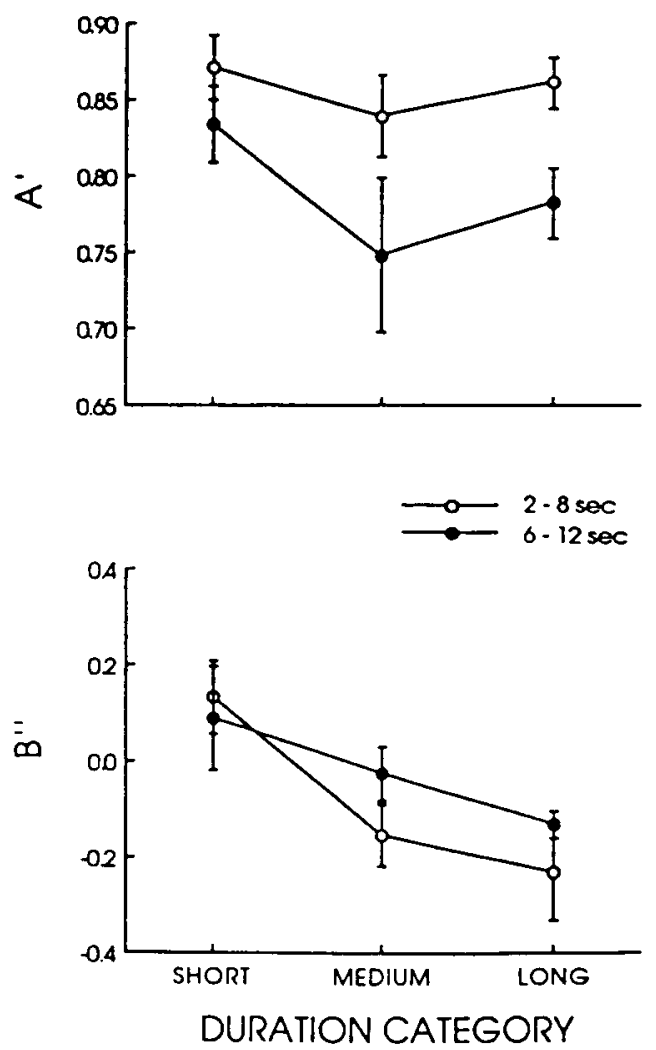

Figure 6. Average sensitivity, $A^{\prime}$, and bias, $B^{n}$, at each of three second-duration categories for performance on the 2-8-sec and 612-sec base ranges. (These measures were calculated from data aggregated over the last 10 sessions of the 2-8-sec range and over the first 10 sessions of the 6-12-sec range. Second-duration categories for the 2-8-sec range correspond to those shown in Figure 3. For the 6-12-sec range, second-duration categories were as follows: short-D2 $\leq 7 \mathrm{sec}$; medium-7 sec $<$ D2 $<11$ sec; long-D2 $\geq$ $11 \mathrm{sec}$. As with the 2-8-sec range, these categories divided the problems roughly into thirds. Error bars show standard errors of the means. Note the restricted range of $A^{\prime}$ values represented on the axis.)

Separate two-way (D2 category $\times$ base range), withinsubject analyses of variance were computed for $A^{\prime}$ and $B^{\prime \prime}$. The analysis for $A^{\prime}$ revealed no significant effects of either variable or of their interaction. The analysis for $B^{\prime \prime}$ showed a significant change in bias over duration category $[F(2,6)=12.97, p<.01]$, but no other significant effects. Although these analyses revealed no significant changes in sensitivity, the high degree of variability in $A^{\prime}$ shown in Figure 6 prompted a closer look at the data. For this analysis, the pigeons were divided into two groups: absolute and relative responders. The pigeons whose performance appeared least affected by absolute duration effects in Figures 3 and 5, B50 and B86, were dubbed relative responders. Pigeons B88 and B96, whose performance in Figures 3 and 5 revealed marked effects of absolute duration, were dubbed absolute responders.

Figure 7 shows average sensitivity, $\mathrm{A}^{\prime}$, and bias, $\mathrm{B}^{\prime \prime}$, for absolute and relative responders over $D 2$ categories for each base range. In both panels, unfilled symbols represent averages over the last 10 sessions of the $2-8-\mathrm{sec}$ base range and filled symbols represent averages over the first 10 sessions of the 6-12-sec base range. Looking first at the upper panel, several points are evident. First, sensitivity for the 6-12-sec base range fell below that of the 2-8-sec range for both absolute and relative responders. Second, absolute responders showed a much larger drop in sensitivity when shifted to the 6-12-sec range than did the relative responders. In the bottom panel, absolute and relative responders show a change in bias over D2 categories under both base ranges. There was, however, a larger change for absolute than for relative responders.

Characterizing one group of pigeons as absolute responders and the other as relative responders should not be taken to imply that the behavior of either group is controlled exclusively by absolute or relative duration. The data in Figures 3 and 5 make it clear that there are degrees of control by absolute and relative factors. The behavior of two of the pigeons, the absolute responders, was simply affected to a greater degree by absolute duration. The small number of subjects prevented statistical verification of the effects shown in Figure 7, but the trends do sug-
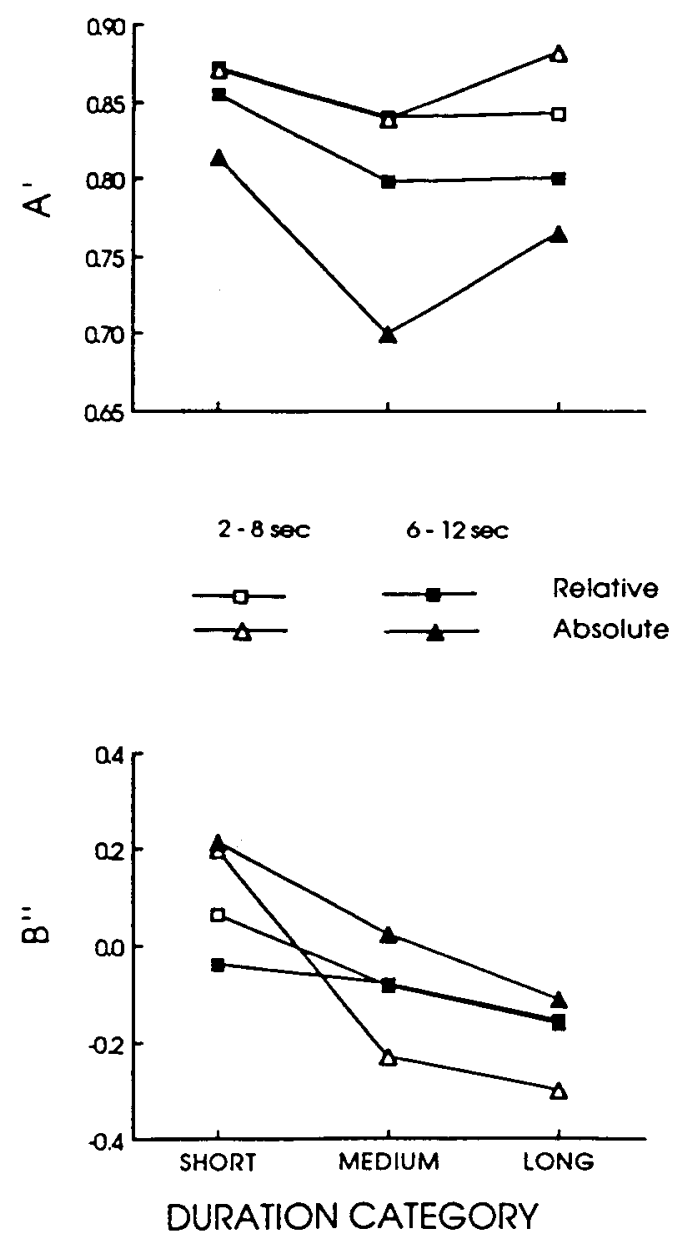

Figure 7. Average sensitivity, $A^{\prime}$, and bias, $B^{\prime \prime}$, at each of three second-duration categories for absolute and relative responders on the 2-8-sec and 6-12-sec base ranges. (Other details as in Figure 6.) 
gest distinctly different patterns of performance for absolute and relative responders, and these different patterns appear to account for much of the variability in performance shown in Figure 6.

\section{DISCUSSION}

Pigeons acquired a discrimination of temporal relations, and their choices were based in part on absolute duration and in part on relative duration. These findings are similar to those of earlier experiments; they are unique, however, in several respects. In this experiment, at least one duration of each pair was unpredictive of relative duration. Accurate performance could therefore occur by comparing durations or by responding on the basis of the absolute duration of a predictive pair member when available. Analyses of performance by D1 and D2 categories showed that when performance was influenced by absolute duration, it was the second rather than the first member of the pair that produced the effect. Therefore, on problems where the D2 of the pair was unpredictive of correct choice, accurate perofrmance could occur only by virtue of control by relative duration. Insofar as accuracy on a subset of problems for which D2 was unpredictive of duration ratio was comparable to that on other problems, the present data demonstrate control of choices by relative duration. These findings, coupled with the fact that overall accuracy exceeded the level that could be achieved by nonrelational means, support the conclusion that there is a relational component of performance on the duration-comparison task.

That performance on this task is controlled in part by absolute duration and in part by relative duration was further illustrated by transfer to a different set of base durations. Performance was significantly poorer on a subset of problems likely to be affected by responding on the basis of absolute duration. In addition, transfer data in Figures 5 and 7, as well as the data in Figure 3, revealed substantial individual differences in the extent to which behavior was controlled by absolute or relative duration. The present data do not make clear what factors contributed to these individual differences in absolute and relational control. Each of the pigeons received the same set of problems in each session, yet there were clear differences in the extent to which absolute duration affected performance. These individual differences in absolute and relative control may present the greatest challenge for models of performance on this task.

The finding that choices were controlled by both absolute and relative duration is consistent with our earlier findings (Dreyfus et al., 1988) and consistent with results from different procedures. For example, Wright, Cook, and Kendrick (1989) trained monkeys on a serial-proberecognition task, a task that nominally requires a response based on the relation between probe and list stimuli, and discovered that over the course of extended training with the same stimuli, behavior came under the control of the absolute properties of the probe stimuli. This absolute con- trol was not exclusive, however; subsequent transfer tests showed that responding remained sensitive to the relation between list items and probes. In the present experiment, control by absolute duration seemed to be primarily by the second member of the duration pair. One obvious reason for greater control by D2 is its relative proximity to the choice response. There is extensive precedent for such a recency phenomenon. In one recent example, Alsop and Honig (1991) trained pigeons to respond on the basis of the relative numerosity of two classes of serially presented stimuli. They found that choice responses varied as a function of relative numerosity but that stimuli occurring later in the series exerted greater control over choices than did earlier stimuli.

Control by stimulus relations in the present experiment contrasts with numerous failures to obtain relational control of pigeon behavior on other nominally relational tasks. For example, the matching-to-sample (MTS) task, often employed in the study of stimulus relations and abstract conceptual behavior, requires that a subject choose which of two or more comparison stimuli matches a sample (i.e., is the same along some dimension). One test of the generality of the behavior produced by this task involves the presentation of novel sample and comparison stimuli. Successful transfer to novel stimuli is taken by some to indicate the presence of an abstract relational concept, that is, sameness. Reviews of the relevant literature generally conclude that there is little evidence for successful transfer by pigeons to novel sample and comparison stimuli (e.g., Premack, 1978; Wright, Cook, Rivera, Sands, \& Delius, 1988). There is no implicit contention that successful discrimination of temporal relations on the paircomparison task requires or involves the presence of abstract relational concepts, but the task is learned with relative ease and performance generalizes to novel duration ratios and novel durations in a way that sets it apart from many attempts to transfer MTS performance.

There are several possible reasons for these differences in performance on duration-comparison and MTS tasks. One salient difference is that the duration-comparison task, especially more recent versions, involves a large number of durations and duration ratios, whereas many studies of MTS involve the presentation of only two or four different stimulus values as sample and comparison stimuli. One exception to this generalization illuminates the potential role of number of stimuli. Wright et al. (1988) trained one group of pigeons on an MTS task using 152 different pictures as sample and comparison stimuli; a second group received a comparable number of trials but with the same two pictures presented on each trial. The performance of the pigeons trained with the larger set of stimuli transferred to novel stimuli, whereas that of the group trained with the smaller set of stimuli did not. Wright et al. suggested that limited transfer to novel stimuli obtained in most MTS studies is less indicative of the limited conceptual abilities of pigeons than of the small set of stimuli employed in training. Results from the earlier version of the duration-comparison task in which fewer durations 
were employed (Fetterman \& Dreyfus, 1986) as well as recent results by Kraemer (1990) reinforce the view that the relatively large set of stimuli used in the current version of the duration-comparison procedure may promote relational learning. (Other differences between the durationcomparison and MTS tasks that may be important are discussed by Dreyfus, 1992.)

Another feature of the duration-comparison task that may influence the development of relational control is the use of stimuli that are unpredictive of duration ratio. Our earliest experiments (Fetterman \& Dreyfus, 1986) not only had fewer stimulus values, but absolute duration was more predictive of relative duration. If predictability is important, we might expect that the lower the predictability of relative by absolute duration, the more extensive the control by stimulus relations. This notion is corroborated by the work of Wright et al. (1989), who attempted to minimize the opportunity for control by absolute properties of probe stimuli under the serial-proberecognition task. These authors found when new stimuli were introduced frequently, the monkeys' behavior remained under the control of the relational properties of the stimuli. Other relevant work by Hulse and his colleagues suggests, however, that both absolute and relative cues are necessary for the subsequent development of relational control. For example, Page, Hulse, and Cynx (1989) trained starlings to discriminate rising from falling sequences of tones. In one set of stimuli, only 8 of the 64 sequences could be identified as rising or falling on the basis of absolute pitch; the remaining sequences required a relative judgment for classification. None of the 7 starlings exposed to these training conditions learned the discrimination. By contrast, another group of starlings was trained with a set of stimuli that included 8 rising or falling sequences of tones for which both absolute and relative pitch cues were relevant (i.e., there was a high correlation between contour, rising or falling, and the initial frequency). When these birds were transferred to the original set of 64 sequences, they were able to discriminate rising from falling sequences, apparently making use of both absolute and relative cues. Although durations unrelated to duration ratio may have promoted control by relative rather than by absolute duration in the current experiment, the results of Page et al. suggest that if there had been no correlation of absolute with relative duration during training, the discrimination may not have developed.

This research began with questions about the relational status of performance on the duration-comparison task. The evidence presented suggests that performance is based on relative duration, but there is also partial control of choices by absolute duration. Although a more categorical answer to this question might be, in some ways, more satisfying, there is an important message in the nature of the response. In general terms, these results should remind us first that behavior is often multiply determined. Behavior that is controlled by one unchanging stimulus or even several stimuli on the same dimension is probably most prevalent in the laboratory. Second, these data should remind us that when behavior is controlled by correlated dimensions (e.g., time and responses, absolute and relative duration), the control by one dimension is rarely exclusive of the other (see, e.g., Fetterman, Stubbs, \& Dreyfus, 1986; Fetterman, Stubbs, \& MacEwen, 1992). Attempts to understand stimulus control in mutually exclusive, unidimensional terms may produce simple questions but will not very likely improve our understanding of complex behavior.

\section{REFERENCES}

ALSOP, B., \& HoNIG, W. K. (1991). Sequential stimuli and relative numerosity discrimination in pigeons. Journal of Experimental Psychology: Animal Behavior Processes, 17, 386-395.

Brown, P. L., \& Jenkins, H. M. (1968). Autoshaping of the pigeon's key-peck. Journal of the Experimental Analysis of Behavior, 11, 1-8.

DREYFus, L. R. (1992). Absolute and relational control in a temporal comparison task. In W. K. Honig \& J. G. Fetterman (Eds.), Cognitive aspects of stimulus control (pp. 21-46). Hillsdale, NJ: Erlbaum.

Dreyfus, L. R., Fetterman, J. G., Smith, L. D., \& Stubbs, D. A. (1988). Discrimination of temporal relations by pigeons. Journal of Experimental Psychology: Animal Behavior Processes, 14, 349-367.

Fetterman, J. G., \& Dreyfus, L. R. (1986). Pair comparison of durations. Behavioural Processes, 12, 111-123.

Fetterman, J. G., \& Dreyfus, L. R. (1987). Duration comparison and the perception of time. In M. L. Commons, J. E. Mazur, J. A. Nevin, \& H. Rachlin (Eds.), Quantitative analyses of behavior: Vol. 5. The effects of delay and intervening events on reinforcement value (pp. 3-27). Hillsdale, NJ: Eribaum.

Fetterman, J. G., Dreyfus, L. R., \& Stubes, D. A. (1989). Discrimination of duration ratios. Journal of Experimental Psychology: Animal Behavior Processes, 15, 253-263.

Fetterman, J. G., Stubbs, D. A., \& Dreyfus, L. R. (1986). Scaling of events spaced in time. Behavioural Processes, 13, 53-68.

Fetterman, J. G., Stubes, D. A., MacEwen, D. (1992). The perception of the extended stimulus. In W. K. Honig \& J. G. Fetterman (Eds.), Cognitive aspects of stimulus control (pp. 1-20). Hillsdale, NI: Erlbaum.

GrIER, J. B. (1971). Nonparametric indices for sensitivity and bias: Computing formulas. Psychological Bulletin, 75, 424-429.

Kraemer, P. J. (1990). Nonrelational processing of a sequential duration discrimination by pigeons. Bulletin of the Psychonomic Society, 28, 71-73.

LAWRence, D. H., \& DeRuvera, J. (1954). Evidence for relational transposition. Joumal of Comparative \& Physiological Psychology, 47, $465-471$.

Olton, D. S., \& SAmuelson, R. J. (1976). Remembrance of places passed: Spatial memory in rats. Journal of Experimental Psychology: Animal Behavior Processes, 2, 97-116.

Page, S. C., Hulse, S. H., \& CYNX, J. (1989). Relative pitch perception in the European starling (Sturnus vulgaris): Further evidence for an elusive phenomenon. Journal of Experimental Psychology: Animal Behavior Processes, 15, 137-146.

Premack, D. (1978). On the abstractness of human concepts: Why it would be difficult to talk to a pigeon. In S. H. Hulse, H. Fowler, \& W. K. Honig (Eds.), Cognitive processes in animal behavior (pp. 423-451). Hillsdale, NJ: Erlbaum.

Premack, D. (1983). The codes of man and beasts. Behavioral \& Brain Sciences, 6, 125-167.

Richelle, M., \& Ljeune, H. (1980). Time in animal behaviour. London: Pergamon.

RoItBLAT, H. L. (1987). Introduction to comparative cognition. New York: Freeman.

Roitblat, H. L., Bever, T. G., terrace, H. S. (Eds.). (1984). Animal cognition. Hillsdale, NJ: Erlbaum.

STEVENS, S. S. (1951). Mathematics, measurement, and psychophysics. 
In S. S. Stevens (Ed.), Handbook of experimental psychology (pp. 149). New York: Wiley.

StuBbs, A. (1968). The discrimination of stimulus duration by pigeons Journal of the Experimental Analysis of Behavior, 11, 223-238.

STUBBS, D. A. (1979). Temporal discrimination and psychophysics. In M. D. Zeiler \& P. Harzem (Eds.), Advances in the analysis of behavior: Vol. 1. Reinforcement and the organization of behavior (pp. 341-369). Chichester, England: Wiley.

WrIGHT, A. A., Cook, R. G., \& Kendrick, D. F. (1989). Relational and absolute stimulus learning by monkeys in a memory task. Journal of the Experimental Analysis of Behavior, 52, 237-248.
Wright, A. A., Cook, R. G., Rivera, J. J., Sands, S. F., \& Delius, J. D. (1988). Concept learning by pigeons: Matching-to-sample with trial-unique video picture stimuli. Animal Learning \& Behavior, 16. 436-444.

Wright, A. A., Santiago, H. C., Sands, S. F., Kendrick, D. F., * Cook, R. G. (1985). Memory processing of serial lists by pigeons, monkeys, and people. Science, 229, 287-289.

(Manuscript received September 23, 1991; revison accepted for publication January 24,1992 .) 